\title{
GCU
}

Glasgow Caledonian

University

University for the Common Good

\section{Gypenosides attenuate retinal degeneration in a zebrafish retinitis pigmentosa model}

Alhasani, Reem Hasaballah; Zhou, Xinzhi; Biswas, Lincoln; Li, Xing; Reilly, James; Zeng, Zhihong; Shu, Xinhua

Published in:

Experimental Eye Research

DOI:

10.1016/j.exer.2020.108291

Publication date:

2020

Document Version

Author accepted manuscript

Link to publication in ResearchOnline

Citation for published version (Harvard):

Alhasani, RH, Zhou, X, Biswas, L, Li, X, Reilly, J, Zeng, Z \& Shu, X 2020, 'Gypenosides attenuate retinal degeneration in a zebrafish retinitis pigmentosa model', Experimental Eye Research, vol. 201, 108291. https://doi.org/10.1016/j.exer.2020.108291

\section{General rights}

Copyright and moral rights for the publications made accessible in the public portal are retained by the authors and/or other copyright owners and it is a condition of accessing publications that users recognise and abide by the legal requirements associated with these rights.

Take down policy

If you believe that this document breaches copyright please view our takedown policy at https://edshare.gcu.ac.uk/id/eprint/5179 for details of how to contact us. 
1 Gypenosides attenuate retinal degeneration in a zebrafish retinitis pigmentosa model

2 Reem Hasaballah Alhasani ${ }^{1,4}$, Xinzhi Zhou ${ }^{1}$, Lincoln Biswas ${ }^{1}$, Xing Li $^{5}$, James Reilly ${ }^{1}$, Zhihong Zeng ${ }^{2, *}$

3 and Xinhua Shu ${ }^{1,3,5 *}$

$4 \quad{ }^{1}$ Department of Biological and Biomedical Sciences, Glasgow Caledonian University, Glasgow G4

$5 \quad$ OBA, United Kingdom

$6{ }^{2}$ College of Biological and Environmental Engineering, Changsha University, Changsha, Hunan 410022, P. R. China

$8{ }^{3}$ Department of Vision Science, Glasgow Caledonian University, Glasgow G4 OBA, United Kingdom

$9 \quad{ }^{4}$ Department of Biology, Faculty of Applied Science, Umm Al-Qura University, Makkah, Saudi

10 Arabia

$11{ }^{5}$ School of Basic Medical Sciences, Shaoyang University, Shaoyang, Hunan 422000, P. R. China

12

13 * Corresponding authors: z20181201@ccsu.edu.cn; Xinhua.Shu@gcu.ac.uk

14

15

16

17

18

19

20

21

22 


\section{Abstract}

Retinitis pigmentosa (RP) is a collection of heterogenous genetic retinal disorders resulting in cumulative retinal deterioration involving progressive loss of photoreceptors and eventually in total blindness. Oxidative stress plays a central role in this photoreceptor loss. Gypenosides (Gyp) are the main functional component isolated from the climbing vine Gynostemma pentaphyllum and have been shown to defend cells against the effects of oxidative stress and inflammation, providing protection in experimentally-induced optic neuritis. The zebrafish model has been used to investigate a range of human diseases. Previously we reported early retinal degeneration in a mutant zebrafish line carrying a point-nonsense mutation in the retinitis pigmentosa GTPase regulator interacting protein 1 (rpgrip1) gene that is mutated in RP patients. The current study investigated the potential protective effects of Gyp against photoreceptor degeneration in the Rpgrip1 deleted zebrafish. Rpgrip1 mutant zebrafish were treated with $5 \mu \mathrm{g} / \mathrm{ml}$ of Gyp in E3 medium from 6 hours post fertilization (hpf) till 1 month post fertilization (mpf). Rpgrip1 mutant zebrafish treated with $5 \mu \mathrm{g} / \mathrm{mL}$ of Gyp showed a significant decrease by $68.41 \%(p=0.0002)$ in photoreceptor cell death compared to that of untreated mutant zebrafish. Expression of antioxidant genes catalase, sod1, sod2, gpx1, gclm, nqo-1 and $n r f-2$ was significantly decreased in rpgrip1 mutant zebrafish eyes by $61.51 \%, 77.40 \%, 60.11 \%, 81.17 \%, 72.07 \%, 78.95 \%$ and $85.42 \%$ (all $p<0.0001$ ), respectively, when compared to that of wildtype zebrafish; superoxide dismutase and catalase activities, and glutathione levels in rpgrip1 mutant zebrafish eyes were significantly decreased by $87.21 \%, 21.55 \%$ and $96.51 \%$ (all $p<0.0001$ ), respectively. There were marked increases in the production of reactive oxygen species (ROS) and malondialdehyde (MDA) by 

of pro-inflammatory cytokines IL-1 $\beta$, IL- 6 and TNF- $\alpha$ was also significantly increased by $150.11 \%$, $267.79 \%$ and $190.72 \%$ (all $p<0.0001$ ), respectively, in rpgrip1 mutant zebrafish eyes, compared to that of wildtype zebrafish. Treatment with Gyp significantly counteracted these effects. This study

49 indicates that Gyp has a potential role in the treatment of RP.

50 Keywords: Retinitis pigmentosa; RPGRIP1; photoreceptor death; zebrafish; Gypenosides

51

52

53

54

55

56

57

58

59

60

61

62

63

64

65

66

67

68

69 


\section{Introduction}

Retinitis pigmentosa (RP, MIM\# 268000) is the term applied to a diverse range of inherited retinal disorders characterised by initial death of rod photoreceptors followed by the death of cone photoreceptors. RP is one of the most common retinal dystrophies, affecting 1 in 3000-4000 people worldwide (Petra-Silva and Linden, 2014; Phelan and Bok, 2000; Raghupathy et al., 2013). Leber congenital amaurosis (LCA), the congenital version of RP, was first recognised and described by Leber (1869). LCA is perhaps the severest form of RP, affecting 1 in 40,000-80,000 worldwide and causing significant visual impairment within one year of birth (Sherwin et al., 2008; Stone, 2007). Characterisation of LCA is based on its clinical features: photophobia, sensory nystagmus, early severe visual loss, absent or reduced electroretinogram (ERG) waveform, and amaurotic pupils (Stone, 2007). Mutations in the Retinitis Pigmentosa GTPase Regulator Interacting Protein 1 (RPGRIP1) gene cause LCA (LCA6) (Dryja et al., 2001; Gerber et al., 2001), juvenile RP (Booij et al., 2005), late onset cone-rod dystrophy (CORD13) in humans (Hameed et al., 2003; Huang et al., 2013; Khan et al., 2013) and cone-rod dystrophy in dogs (Mellersh et al., 2013). Around 5\% of all LCA cases is thought to be caused by mutations in the RPGRIP1 gene (Kumaran et al., 2017). Depletion of RPGRIP1 in human retinal pigment epithelial (RPE) cells causes cilia defects and abnormal cytoskeleton (Patnaik et al., 2018). Loss of RPGRIP1 in mice results in developmental defects in photoreceptor outer segments, mislocalization of rhodopsin, and early retinal degeneration (Won et al. 2009; Zhao et al., 2003). Recently we have characterized a mutant zebrafish line that carries a nonsense homozygous mutation in the rpgrip1 gene. The mutant zebrafish demonstrate defects 
91 in the development of the rod outer segment and in the trafficking of ciliary protein, early retinal

92

degeneration and impaired visual function (Raghupathy et al., 2017).

$$
\text { Gynostemma pentaphyllum (G. pentaphyllum) Makino (Jiaogulan in Chinese) is a }
$$

herbaceous vine plant - more accurately a kind of cucurbitaceous liana (gourd family with woody vines to support its growth) - that is widely used in traditional medicine throughout Asia, including the mountainous regions of Vietnam, Japan, Korea and China. G. pentaphyllum Makino contains a variety of compounds including saponins, carotenoids, chlorophylls, flavonoids, polysaccharides, sterols, amino acids, vitamins and minerals (Razmovski-Naumovski et al., 2005). Gypenosides (Gyp), the dammarane-type saponins extract derived from the G. pentaphyllum (Thunb) Makino, are yellow in colour and bitter tasting, and have anti-diabetic, anti-hyperlipedemic , anti-tumour, anti-oxidative and anti-inflammatory properties (Li et al., 2019). We recently demonstrated that Gyp protected RPE cells from $\mathrm{H}_{2} \mathrm{O}_{2}$-induced oxidative damage and inflammation (Alhasani et al., 2018).

Current therapeutic strategies for RP include gene therapy, cell therapy, retinal prostheses and pharmacological approaches (Dias et al., 2018). High genetic heterogeneity of RP and the complexity of photoreceptor degeneration pathways make RP treatment extraordinarily challenging. Oxidative stress has been implicated in the progression of RP. Treatment with antioxidants has been shown to reduce oxidative damage and preserve photoreceptor function in RP animal models (Komeima et al., 2006; Lee et al., 2011; Shen et al., 2005). There is a close link between oxidative stress and inflammation. Mouse RP models exhibit increased proinflammatory cytokines and chemokines preceding photoreceptor degeneration (Karlstetter et al., 2015; Yoshida 
et al., 2013a; Xun et al., 2019). RP patients also have significantly higher levels of proinflammatory cytokines and chemokines in the vitreous fluid (Yoshida et al., 2013b). Microglial activation has been observed in the retinas of RP patients and RP rodent models and is associated with rod cell death (Gupta et al., 2003; Karlstetter et al., 2015; Zhao et al., 2015). Activated microglia phagocytose pre-apoptotic rod cells and secrete proinflammatory cytokines, which exacerbates rod death in RP mouse models; by contrast, inhibition of microglial activation attenuates rod cell death (Zhao et al., 2015). Antioxidants and anti-inflammatory agents offer a therapeutic option (Guadagni et al., 2015), and so there is a pressing need to identify novel anti-oxidative and anti-inflammation agents for the treatment of RP. In the current study we appraised the therapeutic potential of Gyp for RP by using it to treat rpgrip1 mutant zebrafish and investigating subsequent biochemical and histological changes. We found that Gyp treatment delayed photoreceptor death, increased antioxidant capacity and inhibited endoplasmic reticulum (ER) stress and inflammation.

\section{Materials and methods}

\subsection{Toxicity assay}

All animal work received approval from the UK Home Office with Project licence PPL 70/8697. Gypenosides (Gyp), containing several gypenoside molecules, were purchased from Xi'an Jiatian Biotech Co. Ltd, China with $98 \%$ purity and dissolved in $100 \%$ Dimethyl sulfoxide (DMSO) as a stock $(10 \mathrm{mg} / \mathrm{mL})$. To determine the toxicity of Gyp in zebrafish embryonic stages, toxicity assay was carried out using previously described methods (Scholz et al., 2008). Zebrafish embryos at 6 hours post fertilization (hpf) were treated at $28{ }^{\circ} \mathrm{C}$ in twenty-four-well plates (10 embryos/well) containing $500 \mu \mathrm{l} /$ well of Gyp at concentrations of $0,2.5,5.0,15.0,25.0$ and $50.0 \mu \mathrm{g} / \mathrm{ml}$ in E3 
medium ( $5 \mathrm{mM} \mathrm{NaCl}, 0.17 \mathrm{mM} \mathrm{KCl}, 0.33 \mathrm{mM} \mathrm{CaCl} 2,0.33 \mathrm{mM} \mathrm{MgSO}$ ). Mortality, heartbeat and hatching were recorded at 48, 72, 96 and $120 \mathrm{hpf}$. Heartbeat measurement was performed by putting individual embryos into E3 medium on a glass slide and visually counting ventricle beating (beats per minutes, BPM) under Evos microscope.

\subsection{Zebrafish treatment}

To investigate whether Gyp can inhibit or delay photoreceptor degeneration, rpgrip1 mutant

embryos at $6 \mathrm{hpf}$ were treated with Gyp in E3 medium till one month post fertilization (mpf).

There were 3 groups: wildtype in E3 medium (0.05 \% DMSO), rpgrip1 mutants in E3 medium (0.05\% DMSO), and rpgrip1 mutants treated with Gyp (5 $\mathrm{gg} / \mathrm{mL}$ in 0.05\% DMSO). The treatment was carried out in $60 \mathrm{~mm} \times 15 \mathrm{~mm}$ petri dishes in which the sibling and rpgrip1 mutant embryos (6 hpf) were incubated at $28^{\circ} \mathrm{C}$ in $4 \mathrm{~mL}$ E3 medium with or without Gyp (15 embryos/petri dish in triplicates). After 5 days, the embryos were transferred to 1-litre water tanks (15 fish/tank in 100 $\mathrm{ml} \mathrm{E3} \mathrm{medium)} \mathrm{and} \mathrm{kept} \mathrm{at} 24^{\circ} \mathrm{C}$ in the zebrafish facility for 25 days. During the treatment, fish were fed with ZM-100 fry food and brine shrimp (Zebrafish Management Ltd, UK). E3 medium (0.05\% DMSO) with or without Gyp ( $5 \mu \mathrm{g} / \mathrm{mL}$ ) was changed every two days. After the treatment, zebrafish were killed using Schedule one method and eyes were dissected for further examination.

\subsection{Measurement of reactive oxygen species (ROS)}

Eyes from wildtype, untreated and Gyp-treated rpgrip1 mutant zebrafish at $1 \mathrm{mpf}$ were collected and homogenized using the FastPrep ${ }^{\circledR}-24$ homogenizer (MP Biomedicals, UK) on ice in lysis buffer containing $1 \mathrm{mM} \mathrm{MgCl} 2,20 \mathrm{mM}$ HEPES, $0.32 \mathrm{mM}$ sucrose, and $0.5 \mathrm{mM}$ phenylmethylsulfonyl fluoride (PMSF, pH 7.4). The lysates were centrifuged at $10,000 \mathrm{Xg}$ for 5 minutes at $4^{\circ} \mathrm{C}$ and the supernatants were treated with Dichloro-dihydro-fluorescein Diacetate (DCFH-DA) solution (20 
157 signal was measured using the Fluostar Optima microplate reader (BMG-labtech) at $485 \mathrm{~nm}$

158 (excitation) and $525 \mathrm{~nm}$ (emission). The ROS level was calculated as previously described

159 (Alhasasni et al., 2018).

160

161

162

163

164

165

166

167

168

169

170

17130 minutes in $2 \%$ bovine serum albumin (BSA)/PBS and incubated with primary antibody

172 (anti-rhodopsin, Cat. ab98887, Abcam, 1:500 dilution) in 2\% BSA/PBS at room temperature for 2

173 hours. The sections were washed three times (5 minutes/each) with PBS and then incubated with 174 secondary antibody (goat anti-mouse IgG FITC-conjugated, Cat. F0257, Sigma, UK (1:500 dilution)

175 in $2 \%$ BSA/PBS at room temperature for 1 hour. After washing with PBS five times (5

176 minutes/each), the sections were mounted with Vectashield 4',6-diamidino-2-phenylindole (DAPI)

\subsection{Histology and immunohistochemistry (IHC)}

After one month of treatment, zebrafish eyes were dissected, fixed at $4^{\circ} \mathrm{C}$ for 24 hours in $4 \%$ paraformaldehyde (PFA) solution, then washed with PBS twice, dehydrated with ethanol and embedded in paraffin to make tissue wax blocks for histology; alternatively, the eyes were incubated with $5 \%, 15 \%$ or $30 \%$ sucrose for 2 hours and then embedded in Optimal Cutting Temperature (OCT) compound for cryosections. Eye samples of $8 \mu \mathrm{m}$ thickness were sectioned by microtome or cryotome. For histology, sections were dewaxed and stained with haematoxylin-eosin. For measurement of the photoreceptor layer, five eyes from five individual fish from each group were used, two retinal sections of each eye were selected and images were taken from inferior sides. Thickness of the photoreceptor layer was measured using Image J software at the location $0.4 \mathrm{~mm}$ from the optic nerve head. For IHC, cryosections were blocked for minutes/each), the sections were mounted with Vectashield 4 ',6-diamidino-2-phenylindole (DAPI) 
177 (D9542-Sigma, UK). Images were taken with ZEISS LSM 800 confocal microscopy. To quantify the

178 rhodopsin fluorescence signal, 10 retinal sections from five eyes (two sections/each eye) were

179 collected from the control (wildtype zebrafish), rpgrip1 mutants, or rpgrip1 mutants treated with

180 Gyp. In each section, one region $(10 \mu \mathrm{m} \times 10 \mu \mathrm{m}$, under $\times 400$ magnification $)$ in the superior side

181 ( $0.4 \mathrm{~mm}$ from the optic nerve head) and one region $(10 \mu \mathrm{m} \times 10 \mu \mathrm{m}$, under $\times 400$ magnification $)$ in

182 the inferior side ( $0.4 \mathrm{~mm}$ from the optic nerve head) were chosen for quantification of fluorescent

183 signals with ImageJ software. The signals from both the superior region and the inferior region

184 were averaged for the final calculation.

185 2.5. Gene expression

186 Total RNA was extracted from 20 eyes of wildtype, rpgrip1 mutant, or Gyp-treated rpgrip1 mutant

187 zebrafish at $1 \mathrm{mpf}$ by using the TRIzol reagent (Sigma, UK) according to the manufacturer's

188 guidance. cDNA synthesis was performed using High-Capacity cDNA Reverse Transcription Kit

189 (Applied Biosystems, UK). Quantitative real-time polymerase chain reaction (qRT-PCR) was carried

190 out using Platinum ${ }^{\circledast}$ SYBR $^{\circledast}$ Green QPCR SuperMix-UDG w/ROX kit (ThermoFisher Scientific, UK)

191 following the manufacture's protocol. The primers for qRT-PCR are listed in Table S1. $\beta$-actin was

192 used to normalize mRNA levels of candidate genes.

193 2.6. Measurement of activities of superoxide dismutase (SOD) and catalase (CAT) and

194 quantification of glutathione (GSH) and malondialdehyde (MDA) levels

195 Activities of SOD and CAT and levels of GSH and MDA were measured in zebrafish eye samples

196 using individual commercial kits (STA-340 for SOD, STA-341 for CAT, STA-312 for GSH and STA-330

197 for MDA from Cell Biolabs) following the manufacturer's instructions. 

zebrafish were homogenized in protein lysis buffer (T-PER, Thermo Fisher Scientific UK) using a FastPrep ${ }^{\circledR}-24$ homogenizer (MP Biomedicals) on ice. Concentration of protein samples was

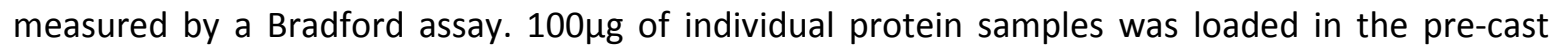
protein gel (Cat. No. 4569034, Bio-Rad) then electrophoresed for $45-60 \mathrm{mins}$ at $200 \mathrm{~V}$ in the running buffer. The gel was removed from the electrophoresis apparatus and assembled into a sandwich containing three sponges, filter paper, pre-cast protein gel, nitrocellulose membrane, filter paper and three more sponges. The sponge-gel-membrane sandwich was then placed into a transfer cassette in the Wet Tank Transfer System (Cat. No. E19051, Thermo Fisher Scientific) containing the transfer buffer to transfer the protein from the pre-cast protein gel onto the nitrocellulose membrane under $175 \mathrm{~mA}$ for 90 minutes. The nitrocellulose membrane was blocked with Tris buffered saline with $0.1 \%$ Tween-20 (TBST) and 5\% (w/v) non-fat dry milk for 2 hours at room temperature. The membrane was incubated with primary antibody (anti-acetylated tubulin antibody, Cat. T7451, Sigma, UK, 1:1000 dilution, anti-Caspase-3 antibody, Cat. ABIN1883652, Antibodies-online, UK, 1:1000 or anti-TNF- $\alpha$ antibody, Cat. ABIN1884227, Antibodies-online, UK, 1:1000 dilution) in TBST with $5 \%(\mathrm{w} / \mathrm{v})$ non-fat dry milk for two hours. After washing three times with TBST, the membrane was incubated with secondary antibody (goat anti-rabbit antibody, Cat. 926-32211 or donkey anti-mouse antibody, Cat. 926-68072, 1:10,000 diluted in TBST with 5\%

217 non-fat dry milk) at room temperature for 2 hours. The membrane was washed five times (5 218 minutes/each). The band intensity of targeted proteins was measured using the LI-COR Odyssey FC 219 Imaging System. 
221 Cell death in zebrafish eye sections was detected using DeadEnd ${ }^{\mathrm{TM}}$ fluorometric TUNEL assay

222 (Promega, UK) according to the manufacturer's instructions. Briefly, following dewaxation

223 zebrafish eye sections were treated with proteinase $\mathrm{K}$ solution $(20 \mu \mathrm{g} / \mathrm{ml})$, then incubated with

224 rTDT reaction at $37^{\circ} \mathrm{C}$ for one hour. The reaction was terminated with $2 \times \mathrm{SSC}$. The sections were

225 mounted with Vectashield DAPI (D9542-Sigma, UK) and the images were taken under ZEISS LSM

226800 confocal microscopy. For quantification of cell death, six sections were chosen from six eyes

227 (one section/each eye) of control, rpgrip1 mutant or Gyp-treated rpgrip1 mutant group; in each

228 section, a region $(50 \mu \mathrm{M} \times 20 \mu \mathrm{M}$, long $\times$ wide $)$ in the superior side $(0.4 \mathrm{~mm}$ from the optic nerve

229 head) was chosen; TUNEL-positive photoreceptor cells in each region were counted and presented

230 as a percentage of the total number of photoreceptor cells in the same region.

231

232

233

234

235

236

237

238

239

240

241

\subsection{Statistical analysis}

Statistical analysis of the data was performed using GraphPad Prism 6 software via one-way ANOVA (analysis of variance) followed by Bonferroni post hoc test. The data were calculated from at least three independent experiments and shown as mean \pm SEM (standard error of the mean). A $p$ value $<0.05$ was considered to be statistically significant.

\section{Results}

\subsection{Toxic effect of Gyp on zebrafish embryos and larvae}

Potential toxicity of Gyp was assessed to allow utilization of a safe dose for subsequent Gyp treatment. Zebrafish embryos at $6 \mathrm{hpf}$ were exposed to Gyp at different concentrations $(2.5,5,15$ and $25 \mu \mathrm{g} / \mathrm{ml}$ ) for 5 days. Treatment with Gyp at 2.5 and $5 \mu \mathrm{g} / \mathrm{ml}$ resulted in mortality over this period that was no different to that of the control group. Higher concentrations, however, caused 
significantly increased mortality: 15 and $25 \mu \mathrm{g} / \mathrm{ml}$ Gyp resulted in, respectively, 30\% and 60\%

243

255 We have shown previously that outer / inner segments of rpgrip1 mutant zebrafish retina were

256 significantly shorter compared to the wildtype retina and that most rod cells had degenerated at

$2571 \mathrm{mpf}$ (Raghupathy et al., 2017). In the current study we performed retinal histology of wildtype,

258 Gyp-treated and untreated rpgrip1 mutant zebrafish at $1 \mathrm{mpf}$ and confirmed that the 259 photoreceptor layer of rpgrip1 mutants was significantly shorter compared to that of wildtype 260 zebrafish. The thickness of the wildtype photoreceptor layer was $\sim 56 \mu \mathrm{m}$, while that of rpgrip1 261 mutant zebrafish was $\sim 36 \mu \mathrm{m}$. Rpgrip1 mutant zebrafish treated with $5 \mu \mathrm{g} / \mathrm{ml}$ Gyp had a 262 significantly thickened photoreceptor layer of $\sim 41 \mu \mathrm{m}$, though the thickness of photoreceptor layer 

Rpgrip1 mutant retinas ((Won et al., 2009). Immunofluorescence staining of retinal sections with 4D2 antibody (detection of rhodopsin) revealed that only a weak signal of mislocalized rhodopsin was present in ONL of zebrafish rpgrip1 mutant retinas, demonstrating early rod degeneration. seen in wildtype zebrafish (Figure 2C).

\subsection{Gyp reduced photoreceptor cell death in rpgrip1 mutant zebrafish}

275 Rpgrip1 mutant zebrafish have been shown to display significant cell death at examined time

276 points: 14 days post fertilization (dpf), $1 \mathrm{mpf}$ and $3 \mathrm{mpf}$ (Raghupathy et al., 2017). In order to

277 examine the protective role of Gyp, we carried out TUNEL assay to assess photoreceptor cell death.

278 We observed significantly increased cell death in the ONL of the mutant zebrafish retinas, around

279 5-fold more compared to the control group. Gyp-exposed rpgrip1 mutant embryos showed a 280 marked decrease in photoreceptor cell death compared to that seen in untreated rpgrip1 mutant 281 zebrafish, while no difference was found between Gyp-treated and wildtype zebrafish (Figure 3). 
process. Relative gene expression analysis using qRT-PCR showed significantly increased expression of caspase3 in rpgrip1 mutant zebrafish eyes compared to those of wildtype zebrafish. Gyp treatment resulted in significantly decreased expression of caspase 3 gene in the mutant zebrafish (Figure S2A). Furthermore, we examined the protein level of Caspase 3 by Western blotting and found that Caspase 3 was significantly increased in rpgrip1 mutant eyes compared to that of wildtype zebrafish, while Gyp treatment significantly counteracted these changes (Figure S2B). When compared to wildtype zebrafish, Gyp-treated mutant zebrafish still had higher expression of Caspase 3 at mRNA and protein levels (Figure S2).

Excessive reactive oxygen species (ROS) production causes cell death through the oxidation and damage of macromolecules. We have shown previously that Gyp can suppress $\mathrm{H}_{2} \mathrm{O}_{2}$-induced ROS production in retinal epithelial cells (Alhasani et al., 2018). Therefore, we conjectured that excessive ROS production underlies increased photoreceptor cell death in rpgrip1 mutant zebrafish retinas and that Gyp might reverse this effect. In fact, ROS production in rpgrip1 mutant zebrafish eyes was $2766 \%$ higher than that in wildtype zebrafish. Treatment of mutant zebrafish with Gyp resulted in a significant decrease in ROS production by $63 \%$ compared to that of untreated rpgrip1 mutant zebrafish eyes; however, even when treated with Gyp, mutant zebrafish had a higher production of ROS than did wildtype zebrafish (Figure 4A). We then assessed the effect of Gyp on antioxidant gene expression using qRT-PCR. We observed that expression of catalase, sod1, sod2, glutathione peroxidase 1 (gpx1), 304 glutamate-cysteine ligase modifier subunit $(g c / m), N A D(P) H$ quinone oxidoreductase-1 (nqo1) and 
NF-E2-related factor-2 ( $n r f 2$ ) genes were markedly decreased in the mutant zebrafish eyes when

compared to that of wildtype zebrafish. Gyp exposure significantly increased the expression of the examined antioxidant genes compared to that of untreated rpgrip1 mutant zebrafish. When compared to wildtype zebrafish, Gyp-treated zebrafish had, with the exception of sod2, a lower expression of the examined genes (Figure 4B-H).

We also measured SOD and catalase activities and the levels of glutathione (GSH) and malondialdehyde (MDA) in the eyes of wildtype, untreated and Gyp-treated rpgrip1 mutant zebrafish. SOD and catalase activities in rpgrip1 mutant zebrafish eyes were significantly decreased when compared to that of wildtype zebrafish; Gyp treatment significantly increased SOD and catalase activities compared to that of untreated rpgrip1 mutant zebrafish, though activities of both enzymes were still lower than that in wildtype zebrafish (Figure 5A, B). GSH level was significantly lower in rpgrip1 mutant zebrafish eyes compared to that of wildtype zebrafish; treatment with Gyp significantly increased GSH level compared to that of untreated rpgrip1 mutant zebrafish; when compared to wildtype zebrafish, Gyp-treated zebrafish eyes had a lower level of GSH (Figure 5C). The level of MDA was higher in rpgrip1 mutant zebrafish eyes compared to wildtype zebrafish, while Gyp treatment resulted in a significantly decreased MDA level compared to that of untreated rpgrip1 mutant zebrafish; compared to wildtype zebrafish, MDA level in Gyp-treated mutant zebrafish eyes was higher (Figure 5D).

\subsection{Protective effect of Gypenoside against ER stress}

ROS production and ER stress are closely related events. ER stress leads to increased transcription

325 and translational responses, thereby causing an increased expression of ER stress inducers (Hetz et 
al., 2011). Accordingly, we examined expression of $x b p 1 t$ (total) and its spliced variant $x b p 1 s$, atf4,

327 atf6 and bip, all of which are induced in ER stress. Expression of all these genes was significantly

328 higher in rpgrip1 mutant zebrafish eyes compared to wildtype zebrafish, while expression was

329 significantly lower in rpgrip1 mutant zebrafish eyes that were treated with Gyp compared to those

330 that were untreated. Expression of atf6, xbp1s and xbp1t (but not atf4 or bip) remained

331 significantly higher in mutant zebrafish treated with Gyp compared to that of wildtype zebrafish

332 (Figure 6).

\subsection{Gyp inhibited inflammation in rpgrip1 mutant zebrafish}

334 We also examined expression of proinflammatory cytokines (il-16, il-6 and tnf- $\alpha$ ) using qRT-PCR and found that expression of these genes was significantly higher in rpgrip1 mutant zebrafish eyes compared to that of wildtype zebrafish; Gyp treatment significantly reduced expression of the three cytokines (Figure 7A). The change of Tnf- $\alpha$ protein level in Gyp-treated rpgrip1 mutant was also confirmed by Western blotting. Expression of the proinflammatory cytokines was higher in

339 Gyp-treated zebrafish eyes compared with that of wildtype zebrafish eyes (Figure 7B).

\section{Discussion}

341 The morphological and functional retinal cell impairment observed in RP is often a result of

342 molecular changes triggered by the retinal damage (Dias et al. 2018). This retinal degeneration

343 might be prompted by several factors including genetic predispositions, higher intraocular pressure, increased blood glucose levels, oxidative/nitrosative stress, or even aging. Irrespective of the trigger factor, a subsequent cascade of cell signals leads to well-established morphological and 
oxidative stress, inflammatory response and induction of apoptotic pathways are characteristic traits in RP (Guadagni et al. 2015).

As a result of the high metabolic activity of photoreceptors, which have the greatest oxygen consumption rate per gram of any body tissue (Ames III, 1992), oxidative stress contributes significantly to the progression of RP (Komeima et al., 2006; Shen et al., 2005; Usui et al., 2009). Numerous animal models of RP suggest that ROS is a stimulus that triggers apoptotic photoreceptor cell death in RP and is aggravated by oxidative stress (Cuenca et al., 2014). Several studies have suggested that the secondary loss of cone cells that is characteristic of RP is a result of damage arising from the higher oxygen consumption and subsequent increased ROS levels when rod cells die (Carmody and Cotter, 2000; Shen et al., 2005). Moreover, ROS production due to light exposure has been demonstrated to exacerbate retinal degeneration in hereditary and age-linked retinopathies including RP (Paskowitz et al., 2006; Sui et al., 2013). Previous studies have demonstrated that Gyp enhance antioxidant capacity, inhibit inflammation and suppress apoptotic cell death in in vivo animal models, such as ischemia/reperfusion injury rodent models (Ye et al., 2016; Yu et al., 2016a; Yu et al. 2016b; Yu et al., 2016c; Zhao et al., 2014). More recently, Gyp have been shown to exhibit protective effects in rodent optic neuritis models by inhibiting lipopolysaccharide (LPS) or myelin oligodendrocyte glycoprotein (MOG) 35-55 peptide-induced inflammation (Wang et al., 2018; Zhang et al., 2017). There are few studies, however, on its role in retinal degeneration. Our previous in vitro study demonstrated that Gyp suppress ROS generation in the ARPE-19 cell line via its effects on gene expression and protein level of several antioxidant scavengers and apoptosis-related genes 
(Alhasani et al., 2018). Our current research has shown that rpgrip1 mutant zebrafish had

significantly higher production of ROS and MDA, lower expression of antioxidant genes, less GSH and high level of rod cell death; Gyp treatment significantly reduced generation of ROS and MDA and increased antioxidant gene expression and GSH level, arresting rod cell degeneration. The NRF2 pathway plays an important role in cellular defence against oxidative damages

373 (Lim et al., 2014). NRF2 is involved in the regulation of several cellular defence mechanisms that counteract the potentially lethal consequences of ROS (Lim et al., 2014). In stress-free conditions NRF2 is localized to the cytoplasm but in the presence of oxidative stress is phosphorylated and

376 translocated to the nucleus, in turn activating several cytoprotective genes (Lim et al., 2014).

377 Activation of the NRF2 functional pathway has been reported to protect against photoreceptor 378 degeneration in RP animal models. Nakagami et al. (2016) demonstrated that NRF2 activation and 379 upregulation of its effector proteins slowed photoreceptor cell death in a RP rabbit model.

380 Adeno-associated virus (AAV)-mediated overexpression of NRF2 in the retina alleviated 381 photoreceptor degeneration in three RP mouse models (Xiong et al., 2015). We too found that rpgrip1 mutant zebrafish eyes had lower expression of NRF2 and its targeting antioxidant genes and that Gyp treatment increased expression of these genes, suggesting that Gyp protection is 384 possibly mediated by the NRF2 signalling pathway. Impairment of protein folding and maturation in ER causes ER stress, leading to the unfolded 386 protein response (UPR). UPR is mediated by protein kinase RNA-like endoplasmic reticulum kinase 387 (PERK), inositol-requiring enzyme 1 (IRE1) and activating transcription factor 6 (ATF6) signalling pathways. ER stress has been implicated in the pathogenesis of RP, while pharmacological 
inhibition of ER stress has been shown to slow retinal degeneration in RP animal models (Dias et al., 2018; Zhang et al., 2014). Previous studies demonstrated that Gyp protected cardiomyocytes

391 from ischemia-reperfusion injury-induced apoptosis via inactivation of C/EBP homologous protein-10 (CHOP) (Yu et al., 2016c). Here we showed that rpgrip1 mutant eyes had higher expression of $a t f 4, a t f 6, x b p 1$ and bip genes, which encode proteins involved in the UPR. Gyp treatment led to significantly decreased expression of these genes, suggesting that Gyp inhibited ER stress that may contribute to decreased rod apoptosis in Gyp-treated rpgrip1 mutant zebrafish. Inflammatory cytokines are overproduced upon exposure to ROS/oxidative stress. Several studies have reported the secretion of various proinflammatory cytokines and chemokines into the vitreous cavity in RP and other ocular diseases, with these cytokines and chemokines being partly responsible for the initiation and development of the condition (Funatsu et al., 2005; demonstrated that deletion of RPGR, an RPGRIP1 interaction protein, caused microglial activation and higher IL-1 $\beta$ expression in mouse retinas (Zhang et al., 2019). In the current study, we also found higher expression of proinflammatory cytokines (il-1 $\beta$, il-6 and tnf- $\alpha$ ) in rpgrip1 mutant eyes and showed that Gyp significantly decreased expression of these genes. In conclusion, we observed that loss of Rpgrip1 in zebrafish resulted in a defective antioxidant defense system and increased ER stress and inflammation. Gyp treatment inhibited

407 oxidative damage, ER stress and inflammation, resulting in slowdown of photoreceptor death in 408 rpgrip1 mutant zebrafish. 
Acknowledgments This study was funded by a Saudi Arabia government PhD scholarship, the Rosetrees Trust, National Eye Research Centre and the Lotus Scholarship Program of Hunan Province, China. X.S. is a Visiting Professor to Shaoyang University.

\section{References}

Alhasani, R.H., Biswas, L., Tohari, A.M., Zhou, X., Reilly, J., He, J.F., Shu, X., 2018. Gypenosides protect retinal pigment epithelium cells from oxidative stress. Food Chem. Toxicol. 112, 76-85.

Ames III, A., 1992. Energy requirements of CNS cells as related to their function and to their

vulnerability to ischemia: a commentary based on studies on retina. Can. J. Physiol. Pharmacol. 70 Suppl, S158-S164.

Booij, J.C., Florijn, R.J., ten Brink, J.B., Loves, W., Meire, F., van Schooneveld, M.J., de Jong, P.T., Bergen, A.A., 2005. Identification of mutations in the AIPL1, CRB1, GUCY2D, RPE65, and RPGRIP1 genes in patients with juvenile retinitis pigmentosa. J. Med. Genet. 42, e67.

Carmody, R.J., Cotter, T.G., 2000. Oxidative stress induces caspase-independent retinal apoptosis in vitro. Cell Death Differ. 7, 282-291.

Cuenca, N., Fernández-Sánchez, L., Campello, L., Maneu, V., De la Villa, P., Lax, P., Pinilla, I., 2014. Cellular responses following retinal injuries and therapeutic approaches for neurodegenerative diseases. Prog. Retin. Eye Res. 43, 17-75.

Dias, M.F., Joo, K., Kemp, J.A., Fialho, S.L., da Silva Cunha, A. Jr., Woo, S.J., Kwon, Y.J., 2018. Molecular genetics and emerging therapies for retinitis pigmentosa: Basic research and clinical perspectives. Prog. Retin. Eye Res. 63, 107-131. 
Dryja, T.P., Adams, S.M., Grimsby, J.L., McGee, T.L., Hong, D.H., Li, T., Andréasson, S., Berson, E.L., 2001. Null RPGRIP1 alleles in patients with Leber congenital amaurosis. Am. J. Hum. Genet. 68, $1295-1298$.

Funatsu, H., Yamashita, H., Noma, H., Mimura, T., Nakamura, S., Sakata, K., Hori, S., 2005. Aqueous humor levels of cytokines are related to vitreous levels and progression of diabetic retinopathy in diabetic patients. Graefes Arch. Clin. Exp. Ophthalmol. 243, 3-8.

Gerber, S., Perrault, I., Hanein, S., Barbet, F., Ducroq, D., Ghazi, I., Martin-Coignard, D., Leowski, C., Homfray, T., Dufier, J.L., 2001. Complete exon-intron structure of the RPGR-interacting protein (RPGRIP1) gene allows the identification of mutations underlying Leber congenital amaurosis. Eur. J. Hum. Genet. 9, 561-571.

Guadagni, V., Novelli, E., Piano, I., Gargini, C., Strettoi, E., 2015. Pharmacological approaches to retinitis pigmentosa: A laboratory perspective. Prog. Retin. Eye Res. 48, 62-81.

Gupta, N., Brown, K.E., Milam, A.H., 2003. Activated microglia in human retinitis pigmentosa, late-onset retinal degeneration, and age-related macular degeneration. Exp. Eye Res., 76, 463-471. Hameed, A., Abid, A., Aziz, A., Ismail, M., Mehdi, S.Q., Khaliq, S., 2003. Evidence of RPGRIP1 gene mutations associated with recessive cone-rod dystrophy. J. Med. Genet. 40, 616-619. Hetz, C., Martinon, F., Rodriguez, D., Glimcher, L.H., 2011. The unfolded protein response: integrating stress signals through the stress sensor IRE1 $\alpha$. Physiol. Rev. 91, 1219-1243.

Huang, L., Zhang, Q., Li, S., Guan, L., Xiao, X., Zhang, J., Jia, X., Sun, W., Zhu, Z., Gao, Y., 2013. Exome sequencing of 47 chinese families with cone-rod dystrophy: mutations in 25 known causative genes. PloS One. 8, e65546. 

microglia: Just bystander or target for therapy? Prog. Retin. Eye Res, 45, 30-57. retinal phenotype in children. Br. J. Ophthalmol. 97, 760-764. model of retinitis pigmentosa. Proc. Natl. Acad. Sci. U S A. 103, 11300-11305. Antioxidative defense mechanisms controlled by Nrf2: state-of the-art and clinical perspectives in neurodegenerative diseases. Arch. Toxicol_ 88, 1773-1786. 
471 Nakagami, Y., Hatano, E., Inoue, T., Yoshida, K., Kondo, M., Terasaki, H., 2016. Cytoprotective

472 effects of a novel Nrf2 activator, RS9, in rhodopsin Pro347Leu rabbits. Curr. Eye Res. 41,

473 1123-1126.

474 Paskowitz, D.M., LaVail, M.M., Duncan, J.L., 2006. Light and inherited retinal degeneration. Br. J.

475 Ophthalmol. 90, 1060-1066.

476 Patnaik, S.R., Zhang, X., Biswas, L., Akhtar, S., Zhou, X., Kusuluri, D.K., Reilly, J., MaySimera, H.,

477 Chalmers, S., McCarron, J.G., Shu, X., 2018. RPGR protein complex regulates proteasome activity

478 and mediates store-operated calcium entry. Oncotarget 9, 23183-23197.

479 Petrs-Silva, H., Linden, R., 2014. Advances in gene therapy technologies to treat retinitis

480 pigmentosa. Clin. Ophthalmol. 8, 127-136.

481 Phelan, J.K.; Bok, D., 2000. A brief review of retinitis pigmentosa and the identified retinitis pigmentosa genes. Mol. Vis. 6, 116-124.

Raghupathy, R.K., McCulloch, D.L., Akhtar, S., Al-mubrad, T.M., Shu, X., 2013. Zebrafish model for the genetic basis of X-linked retinitis pigmentosa. Zebrafish 10, 62-69.

Raghupathy, R.K., Zhang, X., Liu, L., Alhasani, R.H., Biswas, L., Akhtar, S., Pan, L., Moens, C.B., Li, W.,

Liu, M., Kennedy, B.N., Shu, X., 2017. Rpgrip1 is required for rod outer segment development and ciliary protein trafficking in zebrafish. Sci. Rep. 7, 16881. 
491 embryo model in environmental risk assessment-applications beyond acute toxicity testing.

492 Environ. Sci. Pollut. Res. Int. 15, 394-404.

493 Shen, J., Yang, X., Dong, A., Petters, R.M., Peng, Y.W., Wong, F., Campochiaro, P.A., 2005. Oxidative

494 damage is a potential cause of cone cell death in retinitis pigmentosa. J. Cell. Physiol. 203,

$495 \quad 457-464$.

496 Sherwin, J.C., Hewitt, A.W., Ruddle, J.B., Mackey, D.A., 2008. Genetic isolates in ophthalmic

497 diseases. Ophthalmic Genet. 29, 149-161.

498 Stone, E. M., 2007. Leber congenital amaurosis - a model for efficient genetic testing of

499 heterogeneous disorders: LXIV Edward Jackson Memorial Lecture. Am. J. Ophthalmol. 144,

$500 \quad 791-811$.

501 Sui, G.Y., Liu, G.C., Liu, G.Y., Gao, Y.Y., Deng, Y., Wang, W.Y., Tong, S.H., Wang, L., 2013. Is sunlight

502

exposure a risk factor for age-related macular degeneration? A systematic review and

503 meta-analysis. Br. J. Ophthalmol. 97, 389-394.

504 Usui, S., Komeima, K., Lee, S.Y., Jo, Y.J., Ueno, S., Rogers, B.S., Wu, Z., Shen, J., Lu, L., Oveson, B.C.,

505 2009. Increased expression of catalase and superoxide dismutase 2 reduces cone cell death in

506 retinitis pigmentosa. Mol. Ther. 17, 778-786.

507 Wang, F., Dang, Y., Wang, J., Zhou, T., Zhu, Y., 2018. Gypenosides attenuate

508 lipopolysaccharide-induced optic neuritis in rats. Acta Histochem. 120, 340-346. 

2009. RPGRIP1 is essential for normal rod photoreceptor outer segment elaboration and

511 morphogenesis. Hum. Mol. Genet. 18, 4329-4339.

512 Xiong, W., Garfinkel, A.E.M., Li, Y., Benowitz, L.I., Cepko, C.L., 2015. NRF2 promotes neuronal 513 survival in neurodegeneration and acute nerve damage. J. Clin. Invest. 125, 1433-1445.

514 Ye, Q., Zhu, Y.I., Ye, S., Liu, H., She, X., Niu, Y., Ming, Y., 2016. Gypenoside attenuates renal

515 ischemia/reperfusion injury in mice by inhibition of ERK signaling. Exp. Ther. Med. 11, 1499-1505.

516 Yoshida, N., Ikeda, Y., Notomi, S., Ishikawa, K., Murakami, Y., Hisatomi, T., Enaida, H., Ishibashi, T., 517 2013a. Laboratory evidence of sustained chronic inflammatory reaction in retinitis pigmentosa.

518 Ophthalmology 120, e5-12.

Yoshida, N., Ikeda, Y., Notomi, S., Ishikawa, K., Murakami, Y., Hisatomi, T., Enaida, H., Ishibashi, T., 2013b. Clinical evidence of sustained chronic inflammatory reaction in retinitis pigmentosa.

Yoshimura, T., Sonoda, K.H., Sugahara, M., Mochizuki, Y., Enaida, H., Oshima, Y., Ueno, A., Hata, Y., vitreoretinal diseases. PLoS One 4, e8158. alleviate myocardial ischemia-reperfusion injury via attenuation of oxidative stress and preservation of mitochondrial function in rat heart. Cell Stress Chaperones 21, 429-437. Yu, H., Shi, L., Qi, G., Zhao, S., Gao, Y, Li, Y., 2016b. Gypenoside protects cardiomyocytes against ischemia-reperfusion injury via the inhibition of mitogen-activated protein kinase mediated nuclear factor kappa B pathway in vitro and in vivo. Front. Pharmacol. 7, 148. 
531

532

533 and participating in disk morphogenesis. Proc. Natl. Acad. Sci. U S A, 100, 3965-3970.

against myocardial ischemia-reperfusion injury by inhibiting cardiomyocytes apoptosis via

inhibition of CHOP pathway and activation of PI3K/Akt pathway in vivo and in vitro. Cell. Physiol.

Biochem. 39, 123-136.

Zhang X., Shahani U., Reilly J., Shu X., 2019. Disease mechanisms and neuroprotection by tauroursodeoxycholic acid in Rpgr knockout mice. J. Cell. Physiol. 234, 18801-18812.

Zhang, H.K., Ye, Y., Zhao, Z.N., Li, K.J., Du, Y., Hu, Q.M., He, J.F., 2017. Neuroprotective effects of gypenosides in experimental autoimmune optic neuritis. Int. J. Ophthalmol. 10, 541-549.

Zhang, S.X., Sanders, E., Fliesler, S.J. and Wang, J.J., 2014. Endoplasmic reticulum stress and the unfolded protein responses in retinal degeneration. Exp. Eye Res. 125, 30-40.

Zhao, J., Ming, Y., Wan, Q., Ye, S., Xie, S., Zhu, Y., Wang, Y., Zhong, Z., Li, L., Ye, Q., 2014.

Gypenoside attenuates hepatic ischemia/reperfusion injury in mice via antioxidative and anti-apoptotic bioactivities. Exp. Ther. Med. 7, 1388-1392.

Zhao, L., Zabel, M.K., Wang, X., Ma, W., Shah, P., Fariss, R.N., Qian H, Parkhurst, C.N., Gan, W.B., Wong, W.T., 2015. Microglial phagocytosis of living photoreceptors contributes to inherited retinal degeneration. EMBO Mol. Med. 7, 1179-1197.

Zhao, Y., Hong, D.H., Pawlyk, B., Yue, G., Adamian, M., Grynberg, M., Godzik, A. and Li, T., 2003. 

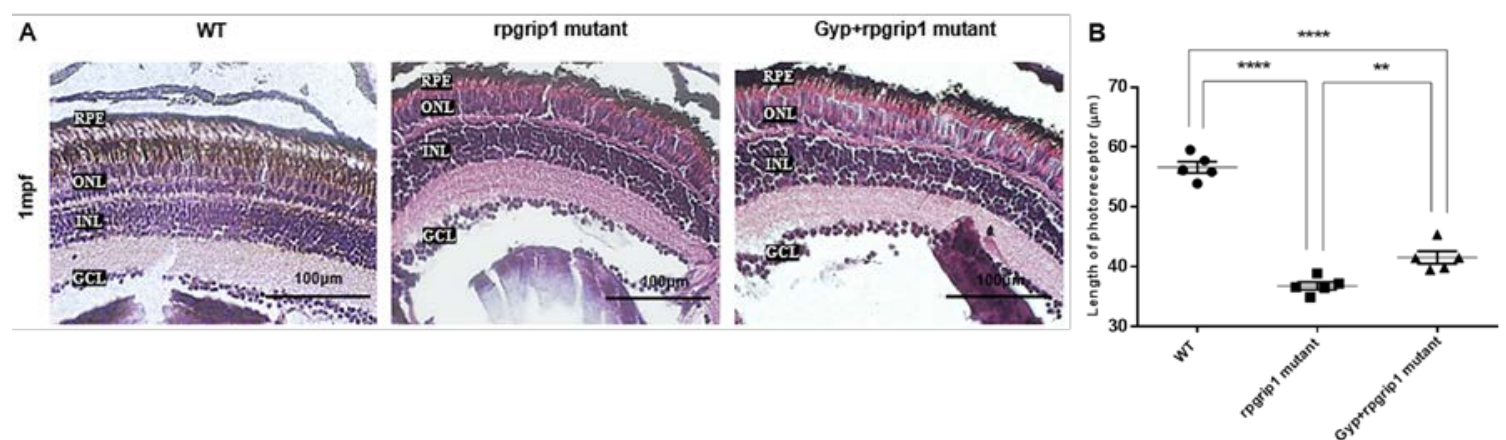

555

556

557

565

566

567

568

569

Figure 1 (A) Representative image of hematoxylin and eosin stained transverse retinal sections of wildtype (WT), untreated (UT) rpgrip1 mutant and Gyp-treated rpgrip1 mutant zebrafish at $1 \mathrm{mph}$. (B) Quantification of the length of photoreceptor cell layer of the examined zebrafish. GCL, ganglion cell layer; INL, inner nuclear layer; ONL, outer nuclear layer; RPE, retinal pigment epithelial cells. The data were analysed using one-way ANOVA followed by appropriate post hoc test (Bonferroni) and presented as mean \pm SEM. ${ }^{* *} p<0.01$ and $* * * * p<0.0001$ 

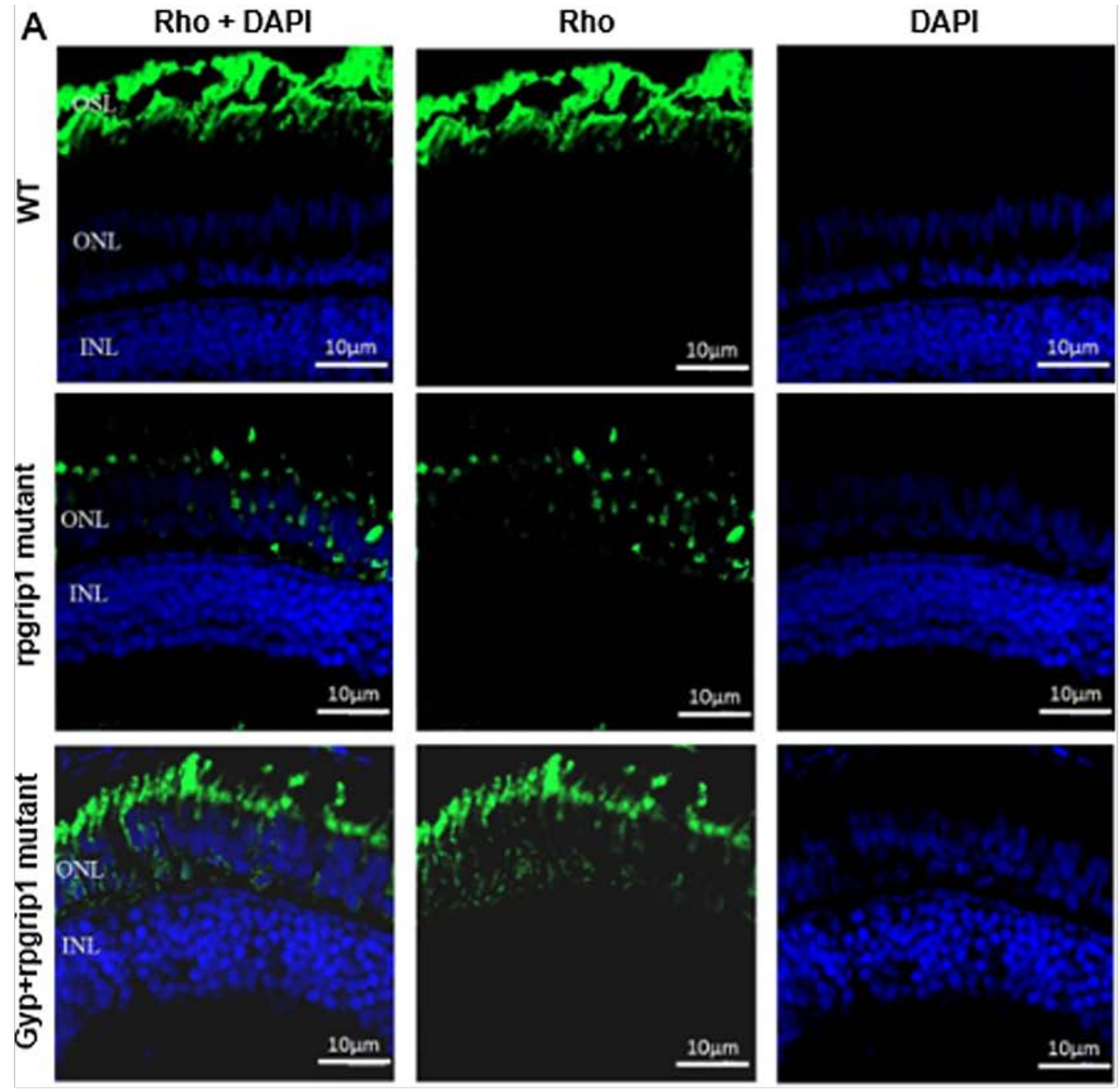

B

C
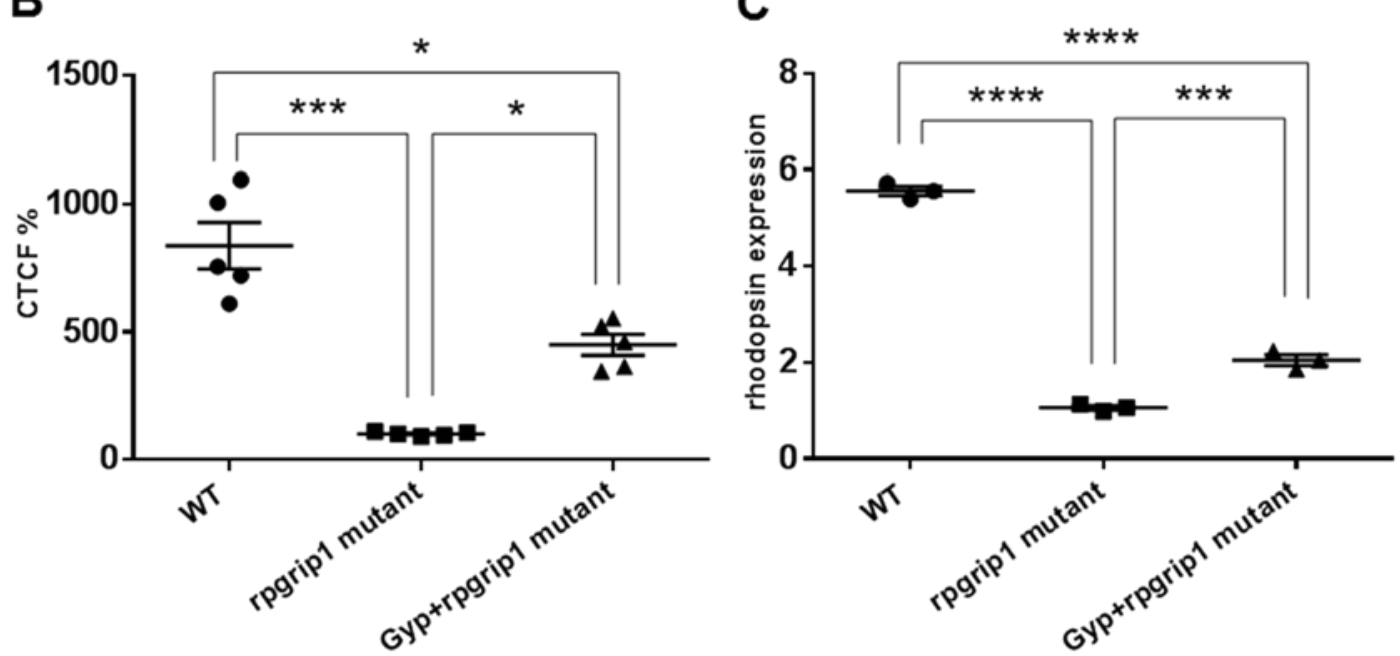

Figure 2 (A) Immunostaining of transverse retinal sections of wildtype (WT), untreated (UT) and Gyp-treated rpgrip1 mutant zebrafish at 1mpf using 4D2 antibody. Rhodopsin localization in the rods was visualized by green fluorescent signals, while nuclei were 

labelled in blue by staining with DAPI. (B) Quantification of the corrected total cell fluorescence (CTCF \%) of rods in retinal sections of examined zebrafish. (C) Expression of rhodopsin was examined by qRT-PCR. INL, inner nuclear layer; ONL, outer nuclear layer. All data were analysed using one-way ANOVA followed by appropriate post hoc test (Bonferroni) and presented as mean \pm SEM. ${ }^{*} p<0.05,{ }^{* * *} p<0.001,{ }^{* * * *} p<0.0001$.

579

580

581

582

583

584

585

586

587

588

589

590 

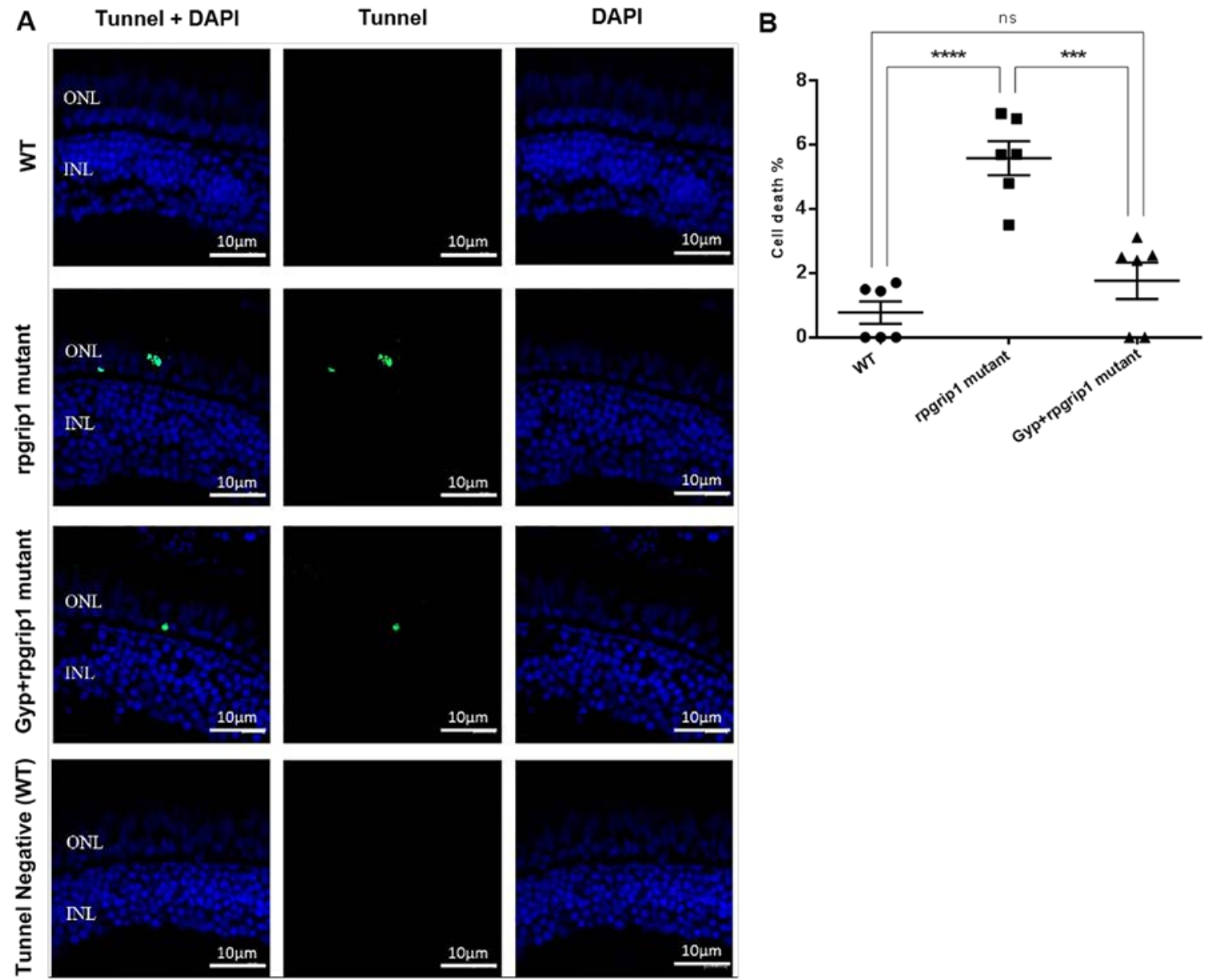

Figure 3 (A) Photoreceptor cell death in wildtype (WT), untreated (UT) and Gyp-treated rpgrip1 mutant zebrafish at $1 \mathrm{mpf}$ retinas was detected using TUNEL assay and photographed by ZEISS LSM 800. (B) The number of cells labelled with positive TUNEL signal was counted within a 50 $\mu \mathrm{m} \times 20 \mu \mathrm{m}$ (long $\times$ wide) area in the outer nuclear layer of the retinal section. The averaged percentage of cells with positive TUNEL signal was counted from six individual animals from each group. A negative control was set up using retinal sections from wildtype zebrafish at $1 \mathrm{mpf}$

598 incubated with the buffer without rTDT. INL, inner nuclear layer; ONL, outer nuclear layer. Data of 599 the fluorescent cells counting were analysed using one-way ANOVA followed by appropriate post 600 hoc test (Bonferroni) and presented as mean \pm SEM. ns, no significance. ${ }^{* * *} P<0.001$ and $* * * * P<$ 601 0.0001 


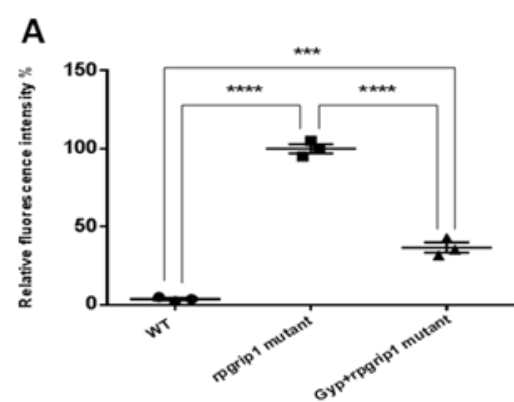

D

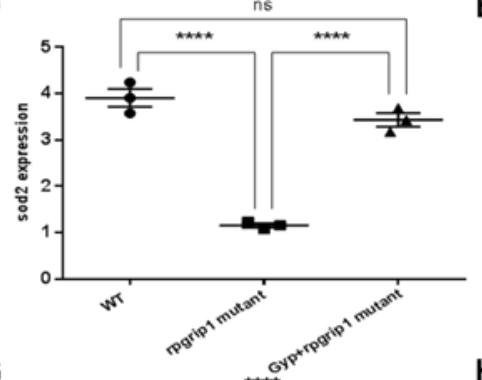

G

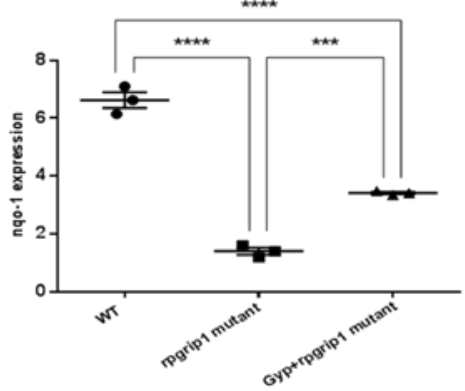

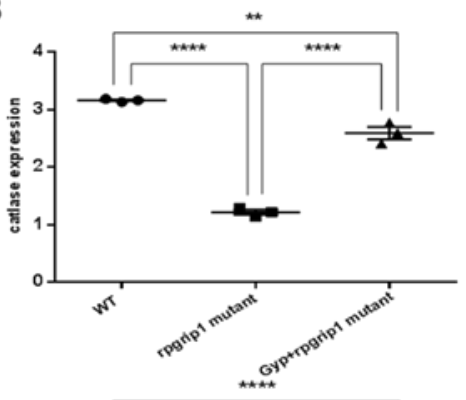

E
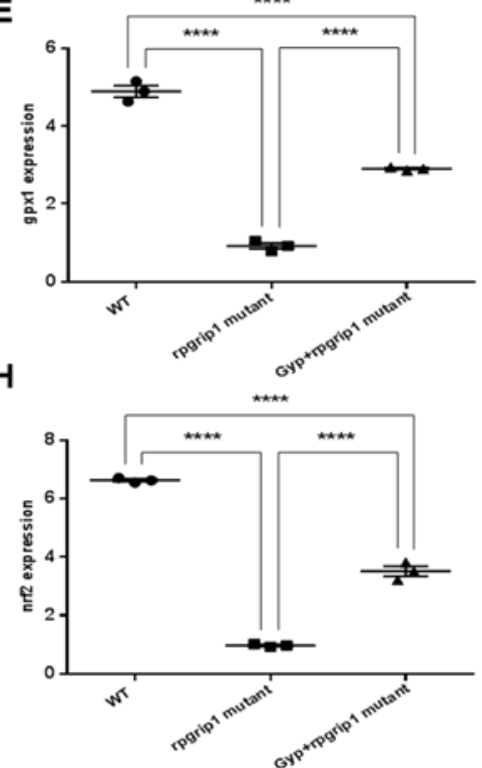

C

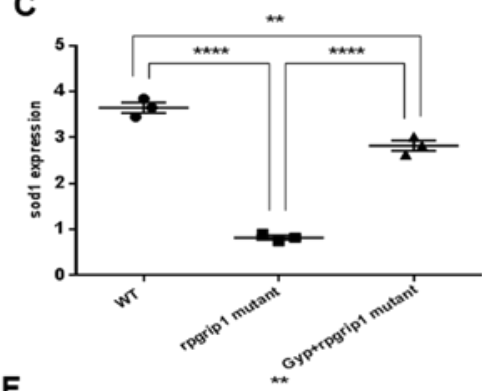

F

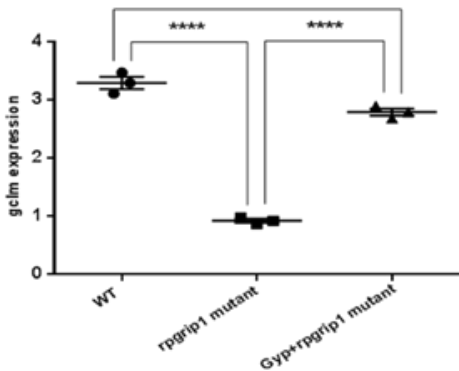

603

604

605
Figure 4. (A) ROS production in eye samples of wildtype (WT), untreated (UT) or Gyp-treated rpgrip1 mutant zebrafish at $1 \mathrm{mpf}$ was measured using DCFH-DA staining described in Materials and methods section. (B-H) Expression of antioxidant genes catalase, sod1, sod2, gpx1, gclm, nqo-1 and nrf2 in the eye samples of wildtype (WT), untreated (UT) or Gyp-treated rpgrip1 mutant zebrafish at $1 \mathrm{mpf}$ was measured by qRT-PCR. All data were analysed using one-way ANOVA followed by appropriate post hoc test (Bonferroni) and presented as mean \pm SEM. $\quad{ }^{*} p<0.05,{ }^{* *} p<0.01,{ }^{* * *} p<0.001$ and $* * * * p<0.0001$ 

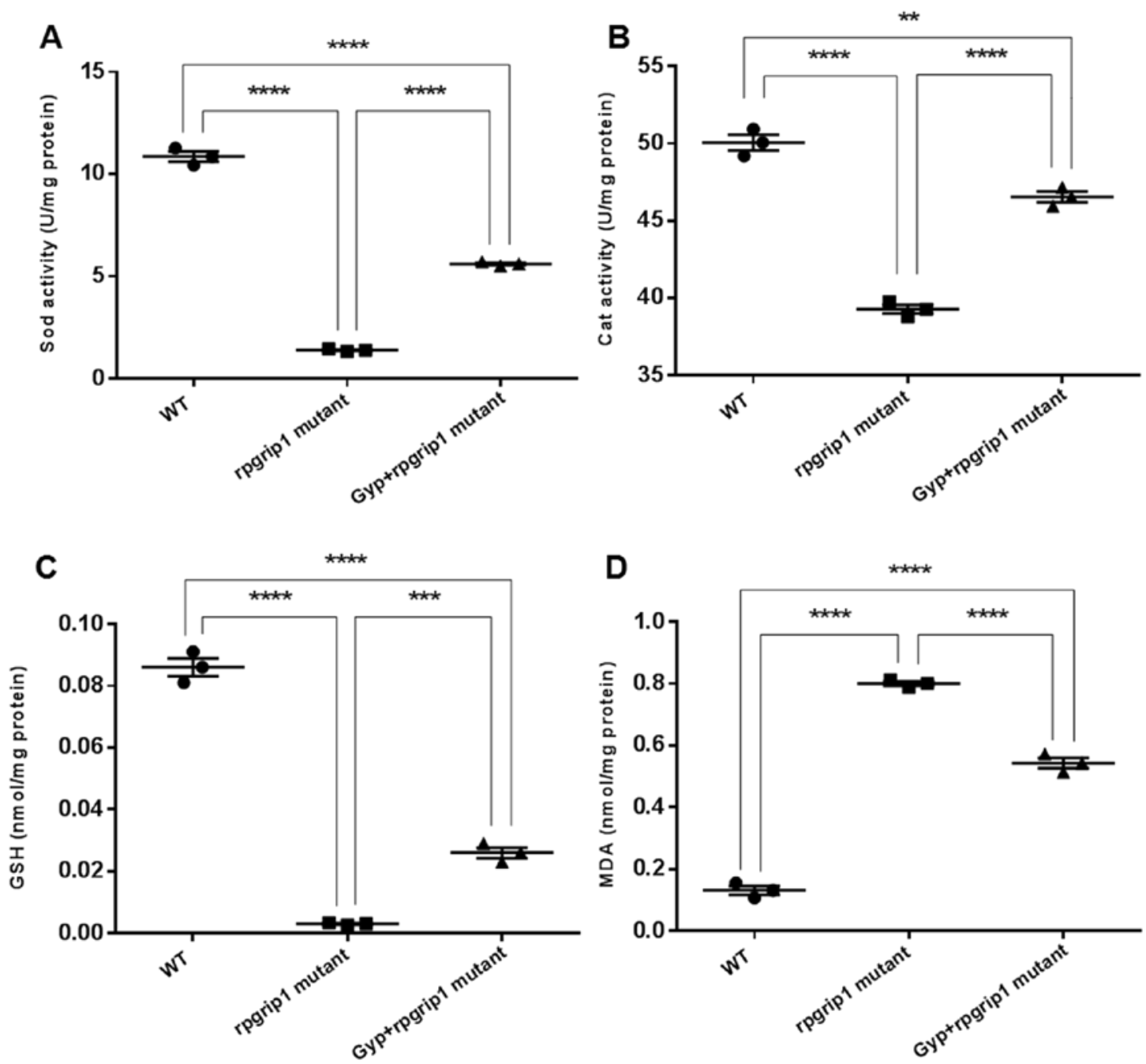

Figure 5. Gyp treatment significantly increased SOD (A) and catalase (B) activities and GSH level (C), decreased MDA level (D) in eye samples of wildtype (WT), untreated (UT) or Gyp-treated rpgrip1 mutant zebrafish at $1 \mathrm{mpf}$. All data were analysed by one-way ANOVA followed by appropriate post hoc test (Bonferroni) and presented as mean \pm SEM. ${ }^{* *} p<0.01,{ }^{* * *} p<0.001,{ }^{* * * *} p<0.0001$ 

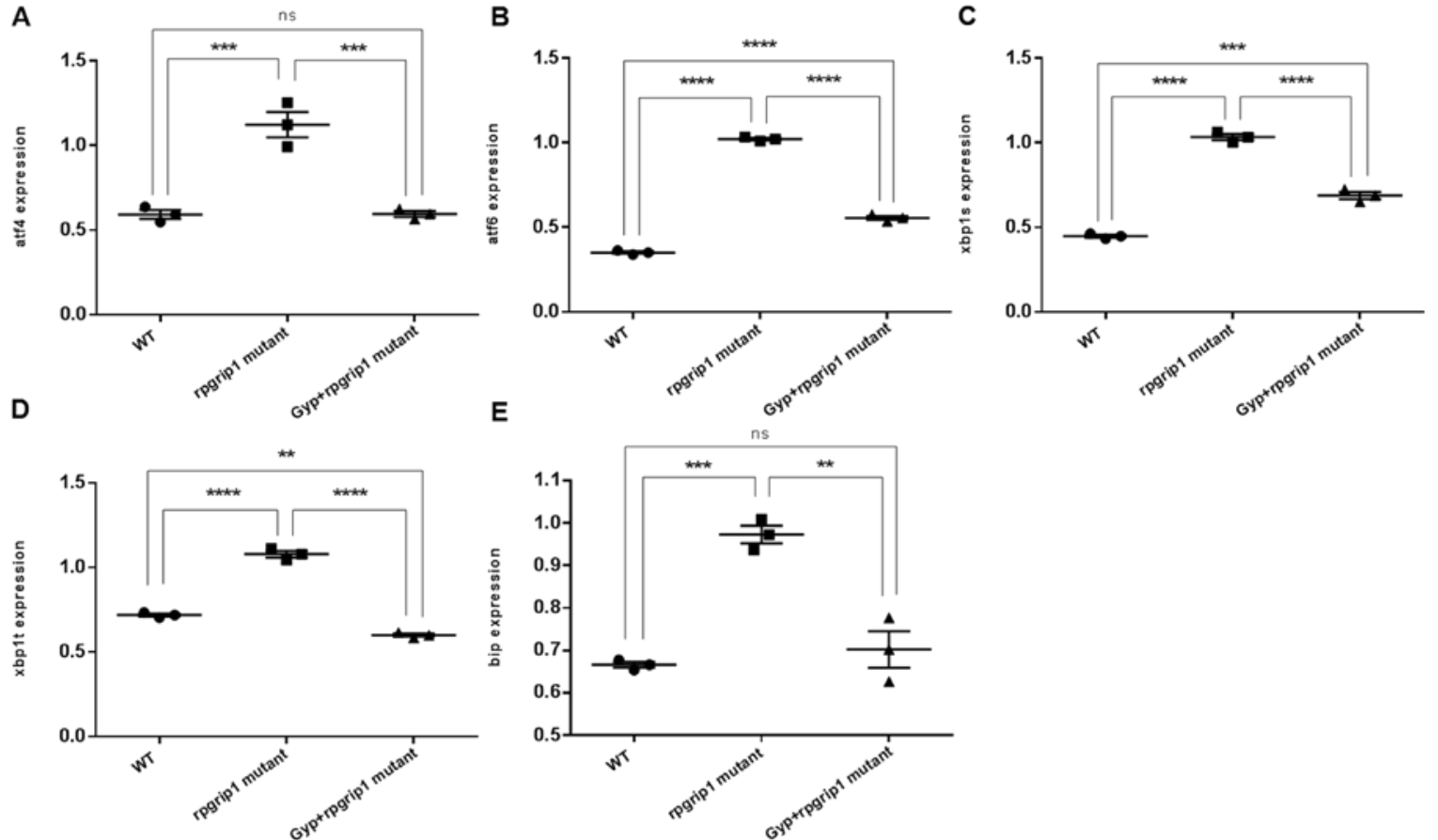

Figure 6. Expression of endoplasmic reticulum (ER) stress-related genes, atf4 (A), atf6 (B), xbp1s (C), xbp1t (D) and bip (E) in eye samples of wildtype (WT), untreated (UT) or Gyp-treated rpgrip1 mutant zebrafish at $1 \mathrm{mpf}$ was measured by qRT-PCR. All data were analysed using one-way ANOVA followed by appropriate post hoc test (Bonferroni) and presented as mean \pm SEM. ns, no significance. ${ }^{* *} p<0.01,{ }^{* * *} p<0.001$ and ${ }^{* * * *} p<$

629 0.0001 .

630

631 

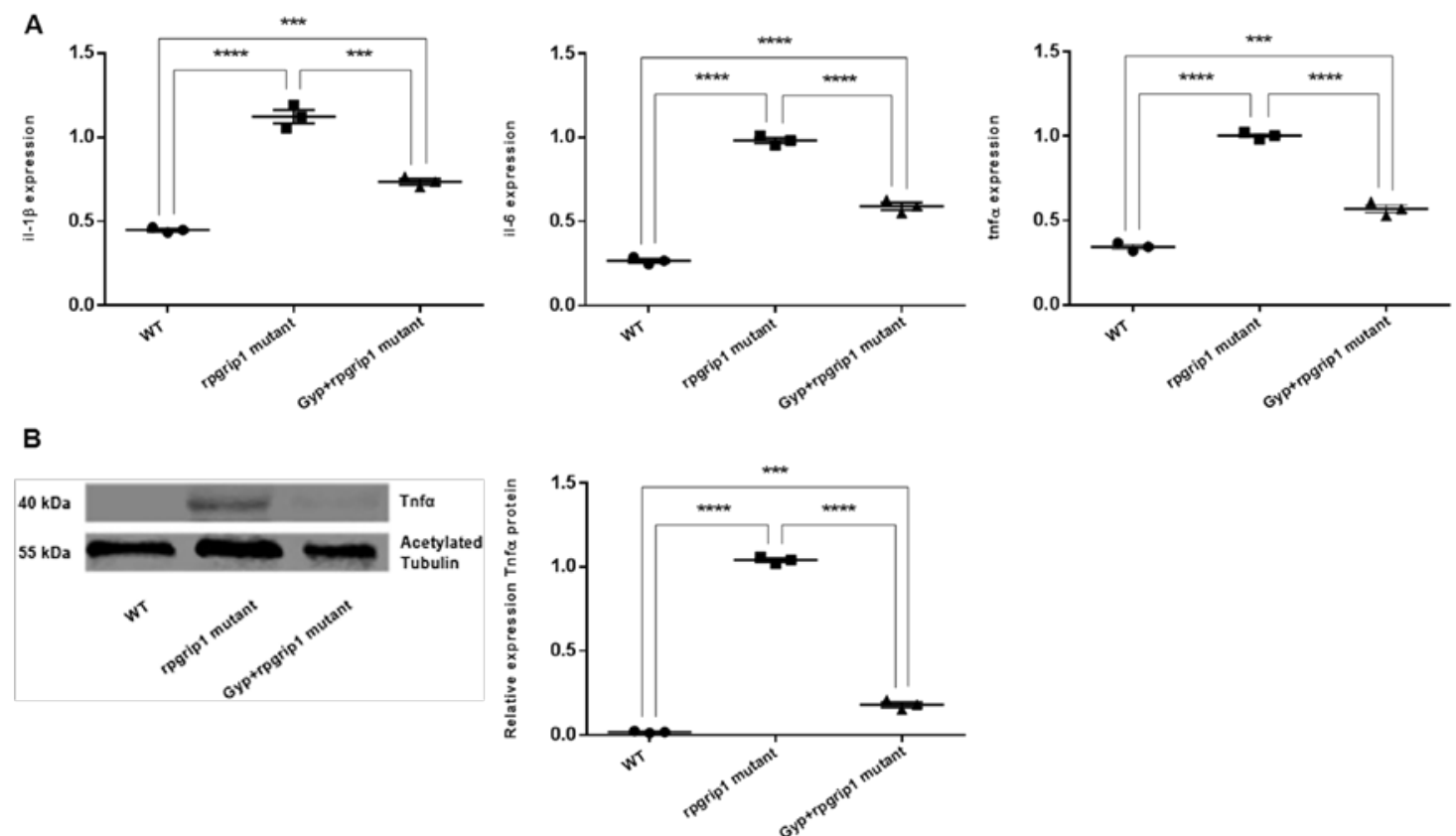

633

Figure 7. Gyp inhibited inflammation in rpgrip1 mutant zebrafish eyes. (A) Expression of il-16, il-6 and tnf- $\alpha$ in eye samples of wildtype, untreated (UT) or Gyp-treated rpgrip1 zebrafish at $1 \mathrm{mpf}$ examined by qRT-PCR. (B) Protein level of Tnf- $\alpha$ in eye samples of wildtype, untreated (UT) or Gyp-treated rpgrip1 mutant zebrafish detected by Western blotting. Quantification of Tnf- $\alpha$ level by normalization of Tnf- $\alpha$ intensity to the intensity of acetylated Tubulin. All data were analysed using one-way ANOVA followed by appropriate post hoc test (Bonferroni) and presented as mean \pm SEM. ${ }^{* * *} p<0.001$, $* * * * P<0.0001$.

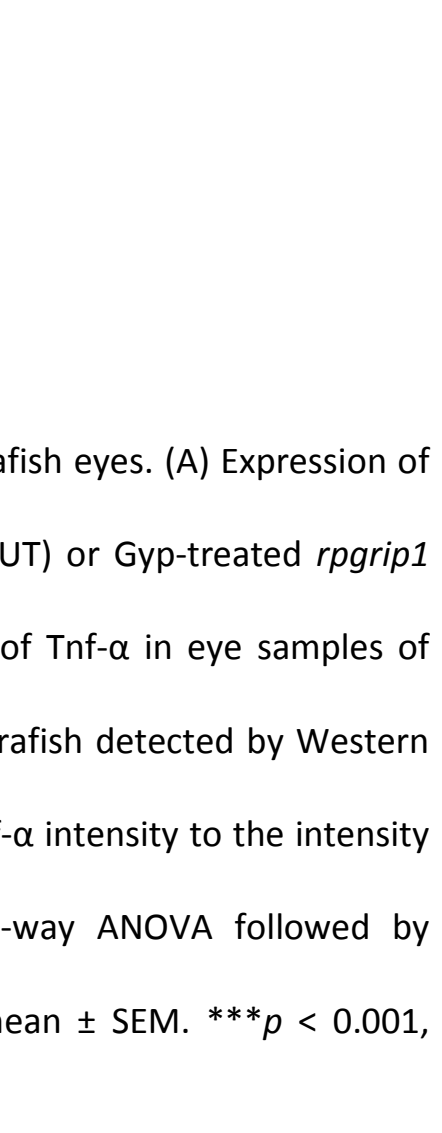

